\title{
PHALANX: Expendable projectile sensor networks for planetary exploration
}

\author{
Michael Dille \\ Moffett Field, CA 94035 \\ michael.dille@nasa.gov \\ Steven McCabe \\ The University of Waikato \\ Hamilton 3216, New Zealand \\ stowme@gmail.com
}

KBR, Inc. / NASA Ames Research Center

\author{
Danny Nuch \\ San Jose State University \\ San Jose, CA 95192 \\ danny.nuch@sjsu.edu
}

\author{
Shiven Gupta \\ Duke University \\ Durham, NC 27708 \\ shiven.gupta@duke.edu \\ Terry Fong \\ NASA Ames Research Center \\ Moffett Field, CA 94035 \\ terry.fong@nasa.gov
}

Nicholas Verzic

Khan Lab School

Mountain View, CA 94041

nicholasverzic@gmail.com

Uland Wong

NASA Ames Research Center

Moffett Field, CA 94035

uland.wong@nasa.gov

\section{Table of Contents}

\begin{abstract}
Technologies enabling long-term, wide-ranging measurement in hard-to-reach areas are a critical need for planetary science inquiry. Phenomena of interest include flows or variations in volatiles, gas composition or concentration, particulate density, or even simply temperature. Improved measurement of these processes enables understanding of exotic geologies and distributions or correlating indicators of trapped water or biological activity. However, such data is often needed in unsafe areas such as caves, lava tubes, or steep ravines not easily reached by current spacecraft and planetary robots.
\end{abstract}

To address this capability gap, we have developed miniaturized, expendable sensors which can be ballistically lobbed from a robotic rover or static lander - or even dropped during a flyover. These projectiles can perform sensing during flight and after anchoring to terrain features. By augmenting exploration systems with these sensors, we can extend situational awareness, perform long-duration monitoring, and reduce utilization of primary mobility resources, all of which are crucial in surface missions. We call the integrated payload that includes a cold gas launcher, smart projectiles, planning software, network discovery, and science sensing: PHALANX.

In this paper, we introduce the mission architecture for PHALANX and describe an exploration concept that pairs projectile sensors with a rover "mothership." Science use cases explored include reconnaissance using ballistic cameras, volatiles detection, and building timelapse maps of temperature and illumination conditions. Strategies to autonomously coordinate constellations of deployed sensors to self-discover and localize with peer ranging (i.e. a "local GPS") are summarized, thus providing communications infrastructure beyond-line-of-sight (BLOS) of the rover. Capabilities were demonstrated through both simulation and physical testing with a terrestrial prototype.

The approach to developing a terrestrial prototype is discussed, including design of the launching mechanism, projectile optimization, micro-electronics fabrication, and sensor selection. Results from early testing and characterization of commercial-off-the-shelf (COTS) components are reported. Nodes were subjected to successful burn-in tests over 48 hours at full logging duty cycle. Integrated field tests were conducted in the Roverscape, a half-acre planetary analog environment at NASA Ames, where we tested up to 10 sensor nodes simultaneously coordinating with an exploration rover. Ranging accuracy has been demonstrated to be within $+/-10 \mathrm{~cm}$ over $20 \mathrm{~m}$ using commodity radios when compared to high-resolution laser scanner ground truthing. Evolution of the design, including progressive miniaturization of the electronics and iterated modifications of the enclosure housing for streamlining and optimized radio performance are described. Finally, lessons learned to date, gaps toward eventual flight mission implementation, and continuing future development plans are discussed.

U.S. Government work not protected by U.S. copyright
1. INTRODUCTION $\ldots \ldots \ldots \ldots \ldots \ldots \ldots \ldots \ldots \ldots \ldots \ldots$

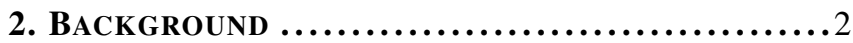

3. HARDWARE APPROACH $\ldots \ldots \ldots \ldots \ldots \ldots \ldots \ldots \ldots$

4. Algorithmic Process ...................6

5. EXPERIMENTATION AND RESULTS .............8

6. Conclusions .............................. 9

ACKnowledgments ........................ 11

REFERENCES .............................. 11

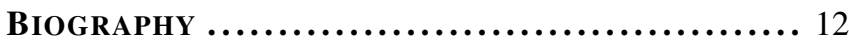

\section{INTRODUCTION}

\section{Motivation}

Future mission expectations present significant challenges to existing planetary surface exploration systems. Targets of scientific interest increasingly lie in or require crossing poorly traversable areas such as steep slopes, rock piles, crevasses, or cliffs that are inaccessible to traditional mission paradigms of a single high-cost rover or lander. Likewise, there is similarly increased desire to dwell in unlit areas such as permanently shadowed lunar regions or operate in dark, poorly mapped, radio-inaccessible subterranean caves and lava tubes, from which a rover must frequently exit for recharging.

Furthermore, science needs increasingly demand richer information streams to build models of macroscopic and often dynamic phenomena, such as climate behavior or gas concentration distribution. The consensus from a recent life detection conference was the need for simultaneous observation across distributed areas and over long durations to avoid the locality pitfalls of prior astrobiology missions [1]. These objectives cannot be fulfilled by a vehicle providing merely a series of sequential single-point measurements, no matter how wellladen a "mobile lab" it may be. Instead, new technologies must be developed to emplace, query, and exploit swarms of science sensors across regions of interest.

Unfortunately, these difficulties are not expected to see a simple resolution. Mobility risk-taking is heavily precluded by the conservative driving style of existing rovers, which have a history of wheel damage and embedding [2]. Upcoming commercial payload programs of reduced scale necessitate smaller, less-equipped rovers. This is part of a broader move 


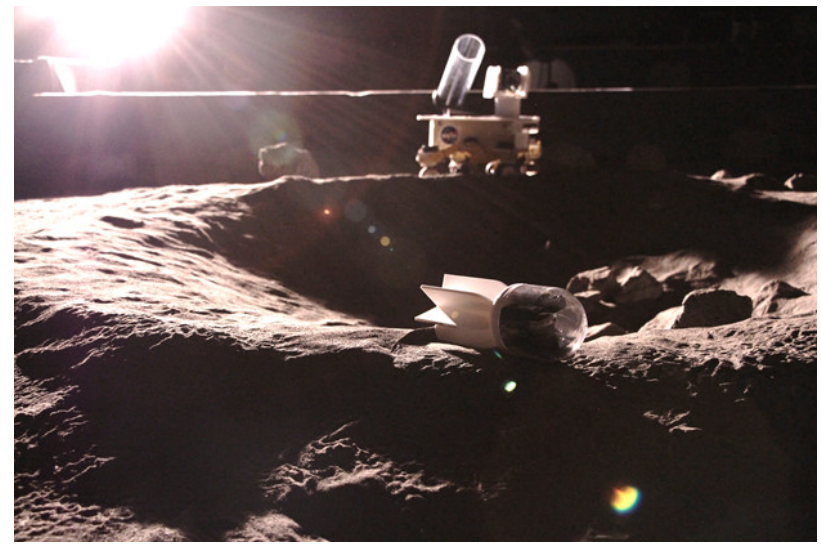

Figure 1. Projectile prototype in lunar simulant sandbox.

towards multi-vehicle systems of heterogeneous teams, in which several daughtercraft explore in tandem, yet each have more limited navigation sensing and communication ability. Finally, many future missions to new bodies will first consist of stationary landers providing no mobility beyond perhaps an articulating robotic arm for sampling.

Numerous exotic mobility concepts have been proposed to overcome such limitations, but these high-risk complex designs remain immature and present high cost. Rovers, with their innate stability and payload economy, will remain the principal explorers for the foreseeable future.

\section{PHALANX Concept}

We have developed an exploration concept, PHALANX (Projectile Hordes for Advanced Long-term and Networked eXploration), utilizing expendable projectile sensors that can be lobbed or rolled into place from a mortar-like delivery mechanism. The key idea is that the launching system can be carried as a payload to extend the reach and monitoring capability of traditional mobile robotic vehicles.

This work builds on a single-sensor concept to develop selforganizing swarms incorporating distributed algorithms for data storage and forwarding, network failure resilience, relative location state estimation, and dense topological science data map generation. This permits existing robotic missions to take calculated sensing risks using massively redundant, expendable hardware.

To illustrate the concept, consider two concepts of operation relevant to distinct planetary environments.

Surface operations-A rover approaches inaccessible points of interest or long-durations sampling target zones, and aims and deploys projectiles towards each. The rover continues driving between such points, repeating the deployment. Once emplaced, nearby nodes form a mesh network, communicating with a fixed node built into the rover. Based on initial data returned and observed communication quality, locations of additional sampling sites may be chosen adaptively. The rover continues its primary science mission, taking it possibly well out of communications range of the network. Opportunistically, the rover may use nodes as localization landmarks, either for accurately returning to a location for repeated sampling, or to augment a terrain slip detection or characterization system. Depending on the particular sensor payload and programmed sampling frequency, nodes can remain emplaced and recording for up to several weeks. As mission planning permits, the rover revisits connected node clusters to retrieve data, requiring proximity to only one per cluster, as the mesh relays data from more distant nodes.

Subterranean reconnaissance-A team of small rovers enters a treacherous previously-unmapped underground cavern. Nodes are released at intervals to augment navigation sensing in low-visibility conditions, possibly as optical or radio localization beacons. Alternatively, specific nodes could provide illumination (allowing photometric stereo reconstruction of the environment from cameras onboard the vehicles) or external camera views by being launched down corridors or by attaching to walls. Projectiles may be launched over non-traversable rock piles to gain insight about the area beyond, potentially exploring further if equipped with a small mobility mechanism themselves, as payload limits may allow. Crucially, a chain of emplaced nodes reaching back to the entrance of the cave offers a system of relay communication back to the outside, where a parent vehicle or lander can return data to Earth. As the rover team will most likely be battery powered and thus require solar-derived recharging outside the cave, they must move quickly to complete their mission and exit frequently. In their stead, emplaced nodes can supply highly desirable long-term environmental monitoring for biosignatures.

This article outlines the development of the PHALANX concept, detailing the hardware prototyping process, explaining techniques for node location selection and data processing, and presents early performance characterization and field data evaluation. Applied to future planetary missions, PHALANX can effectively meet the joint goals of a sensor system with low cost, low risk, and compactness while also maintaining sensing breadth, reach, and redundancy.

\section{BACKGROUND}

In an attempt to address the well-recognized mobility challenges planetary exploration vehicles face, varied mobility concepts have been proposed, including legged walkers [3], hoppers [4], climbers [5], and snakes [6]. These suffer from a need for complex control strategies and are sensitive to unstructured terrain, risking the entire vehicle due to a fall. Rotorcraft will soon see first use via the planned Mars Helicopter Scout [7] but struggle in thin atmospheres, are irrelevant for airless bodies, and present risk in cramped underground environments. A further consideration is socalled planetary protection, for which any propulsive vehicles score poorly due to possible contamination of pristine environments. In contrast, small self-contained projectiles can avoid such contamination, while reducing risk through redundancy and expendability.

In diverse contexts, projectile or ballistic sensors have been considered as an alternative to powered mobility. NASA has previously considered projectiles for multi-kilometer payload delivery, but abandoned the idea in favor of maturing precision lander technology. In terrestrial settings, ballistic devices provide a simple form of standoff sensing that has proved to be particularly appealing in defense and law enforcement applications, where a small package can be lobbed into a possibly dangerous area to perform passive reconnaissance. Based on techniques to generate omnidirectional panoramas [8] from a multi-camera-studded sphere in flight or rolling on the ground, a great many commercial offerings now provide emphasis across the spectrum from 
ruggedness [9] to highest imaging resolution [10]. In more stationary circumstances, spherical cameras may be used for recording scenes for immersive virtual reality applications.

Naturally, including mobility in remotely deployable sensors provides additional benefits. From early work [11] on inherently self-righting highly-survivable mechanisms, many such "throwable robots" are now available as small wheeled or tracked mobile camera platforms. Specialized designs incorporating microspines enable climbing of vertical rocky surfaces [12], while highly compliant structures permit landings from even terminal velocity [13]. In planetary exploration contexts, the proposed PUFFER (Pop-Up Flat Folding Explorer Robot) system demonstrates a means to expand and deploy a tightly packaged nanorover, albeit with limited mobility [14].

Somewhat similarly, dropsondes - devices dropped from aircraft and slowed by a parachute - are commonly used in weather reconnaissance, carrying various atmospheric sensors. These are a special case of radiosondes, general airborne instruments, that are more typically carried by balloon. The Soviet Vega Venus probes are so far the only such balloon sensors successfully flown on a spaceflight mission, though a number of others have since been proposed.

Data collection from multiple sensors positioned around an area forming a sensor network, either recording independently or communicating with one another, provides a more detailed picture of the state and evolution of an environment. This has gained great popularity in the natural sciences, particularly for the study of microclimates. To date however, these are almost universally manually placed, limiting their applicability and reach. Cave environments, of particular interest as planetary analogues and previously commonly thought to present a largely static atmosphere, have recently been shown to exhibit sometimes highly dynamic microclimates through so-called "cave breathing" through sensor network studies requiring often arduous scrambling for sensor emplacement [15].

Sensor networks are similarly immensely useful in outdoor agriculture, particularly for sensitive and high-value crops such as vineyards, where conditions such as leaf wetness may be monitored, transmitting data through multi-hop communications to a base station, from which data may be uploaded to the Internet. By understanding environmental variables at play, farmers can better understand the underlying dynamics of their crops, review of long-term trends, and best select actions (such as pruning, irrigation, or fertilization) maximizing crop health and yield [16].

In the commercial and consumer domains, the "Internet of Things" (IoT) continues to rapidly evolve thanks to extensive progress in sensor miniaturization and networking that is seeing use in home automation, factory process control, and public health monitoring. Technologies and protocols such as WiFi, Bluetooth, LoRa, and Zigbee-along with many readily-available low-cost commercial hardware modulesprovide varied tradeoffs in power consumption, bandwidth, range, and feature sets appropriate for different applications. Meanwhile, progressive improvements in physical sensors, particularly those containing micro-electro-mechanical systems (MEMS) components, have made practical unprecedented low-cost and low-power highly miniaturized devices. Coupled further with ever-advancing processor and flash storage options, affordable networked sensors are easily available for even the most trivial of consumer applications.
However, little effort has been made to apply such advances to planetary exploration. Likewise, the extensive prior research in sensor networks, distributed algorithms, and systems theory has yet to provide unified solutions to the nuanced challenges of planetary scenarios, which include: varying types of node failures; beyond line-of-sight and long-distance communication; extremely limited processing, memory, and battery capacity; terrain map uncertainty; finite sensor count requiring deliberate placement planning; and data flow to a mothercraft (e.g. rover).

\section{HARDWARE APPROACH}

\section{System Overview}

The high-level aim of PHALANX is to create a robust swarm of expendable sensing projectiles that can be deployed in high risk areas to extend the reach and otherwise augment the operation of robotic explorers.

An overall list of potential capabilities therefore includes:

- Imaging - in-flight or post-landing

- Illumination - in-flight or post-landing

- Environmental sensing - local atmospheric, surface, or slightly subsurface

- Communications relay - between nodes or for other agents

- Localization landmarks - beacons locating nodes or mobile robots

The typical scenario of focus herein is a deployment to track environmental conditions in a wide region over time. To achieve this, the nodes must be capable of self-localization with little interaction from the rover/lander. Then, they must survey relevant environmental and atmospheric conditions over time and interpolate the sensing data in-between nodes.

\section{Terrestrial Prototype Development}

The prototype PHALANX system consists of three major parts: the launcher, the projectile nodes (containing processing electronics, communications transceivers, and sensors), and the mobile robotic rover. We will now discuss the specific components of the terrestrial prototype.

Launcher-A spectrum of propulsion means were evaluated. Springs or elastics require a number of moving parts considered risky in flight missions, are temperature sensitive, and necessitate a re-tensioning mechanism for repeated launching. Electromagnetic propulsion (such as a coil or rail gun) presents some appeal due to its need for few moving parts and non-reliance on external fuel, however the large volume of support electronics (e.g. capacitors), relatively weak thrust, and overhead of inert conductive projectile segments negates any benefits. Cold (compressed) gas, specifically carbon dioxide $\left(\mathrm{CO}_{2}\right)$ was selected as the propulsive source due to its simplicity, compactness, and ready availability. In an eventual flight mission it is likely that a small pyrotechnic will be preferable, as the greatly reduced vapor pressure of gases at low (or even simply highly variable) planetary surface temperatures result in much weakened (or inconsistent) thrust. However, due to regulatory and safety constraints, pyrotechnics were impractical for prototyping.

Our prototype launcher is based on a commercial muzzleloaded $\mathrm{CO}_{2}$ mortar that fills a chamber up to a desired pressure from a standard miniature paintball tank (providing 
gas at approximately 5.5MPa at room temperature), and upon triggering, releases the contents of the chamber rapidly into the barrel behind the projectile. Range may be varied, albeit coarsely, up to roughly 30 meters by adjusting the chamber pressure attained up to approximately $1.7 \mathrm{MPa}$ ). Aim is achieved through a custom tilt mechanism that controls elevation angle and by yawing the robot to change heading. The barrel diameter of the launcher is approximately $75 \mathrm{~mm}$. The launcher mechanism as mounted on our analogue test rover is shown in Figure 2.

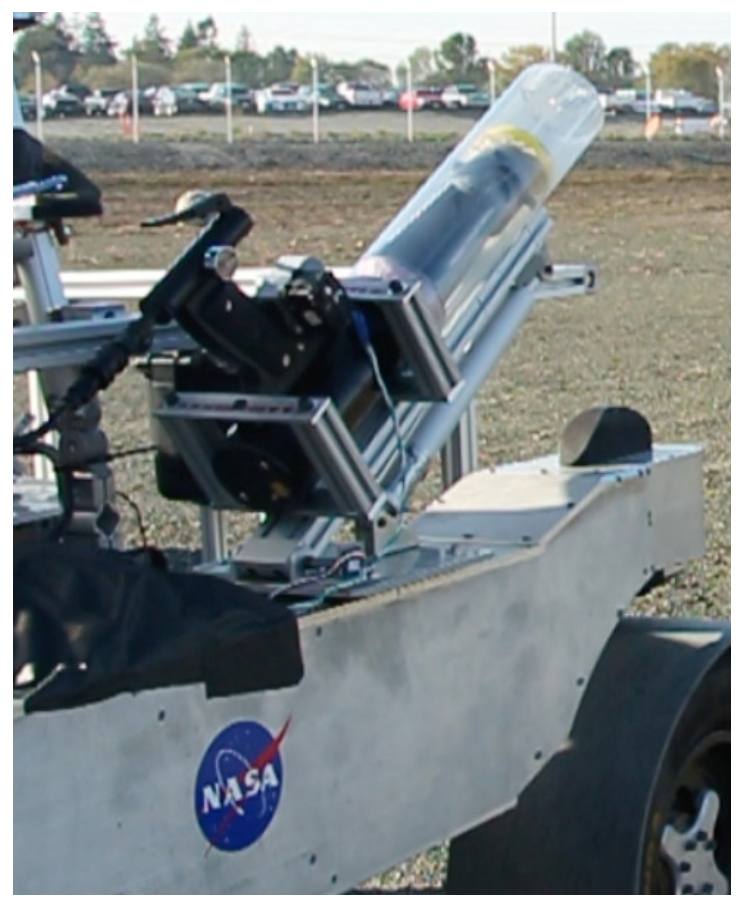

Figure 2. Cold gas propelled projectile launcher.

At this time, the launcher is only capable of firing a single projectile at a time without intervention due to its muzzleloaded design. In the future, we will implement one of several preliminary designs for a feeder mechanism for more relevant breech loading, enabling the sequential launch of swarms of sensing projectiles.

Projectile-Each expendable projectile contains a fully selfcontained sensing node and consists of a ballistic shell, a miniature electronic control board, and modularized sensors. Starting from a simple rectangular enclosure in earliest development, an iterated series of housing designs have been evaluated. The most recent ballistic shell is comprised of a machined aluminum penetrator nose, a shock-mounted electronics sled, and spring-loaded fins that open outward once in flight for additional stability. This shell is approximately $47 \mathrm{~mm}$ in maximum diameter, and a discarding sabot is used for alignment within the barrel of the launcher.

An aerodynamic projectile is of course of reduced or little benefit on low-atmosphere or airless planetary bodies, and indeed, very early experimentation involved spherical enclosures. However, we quickly observed that a spherical shape presents a number of downsides, as such projectiles do not pack efficiently in a given volume, and payloads of interest (e.g. batteries and electronics) are rarely spherically symmetric, resulting in wasted space. Further, an elongated projectile allows stabilized deterministic landing (rather than rolling), greater opportunity for irregular mass distribution, and vertical standoff above the surface for antennas.

Processing electronics - A main custom printed circuit board (PCB) acts as the local hub within each projectile, connecting a processor, flash storage, radio transceivers, and varied sensor daughterboards. It features an Arm Cortex-M4 microcontroller, power protection and regulation circuitry, several connectors for modular sensor attachment, and external pin headers for programming and diagnostics. Like the projectile enclosure, this has undergone several iterations, including a relatively large intermediate variant spanning $50 \mathrm{~cm}^{2}$ and most recently version reduced to $30 \times 65 \mathrm{~mm}$ (including antennas) shown in Figure 3.

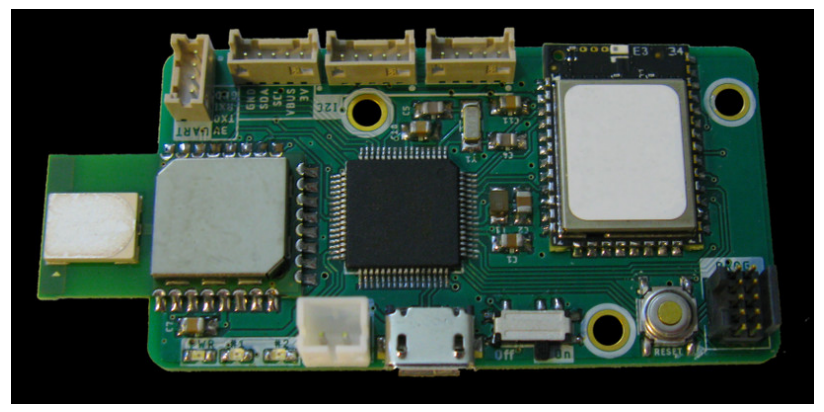

Figure 3. Primary electronics PCB in sensor nodes.

The design of this PCB presents a compromise between the three extremes of a fully relevant flight design, a maximally miniaturized consumer commercial device, and a hobby-level rapid prototype built from disparate $\mathrm{PCB}$-mounted modules. Any flight mission will impose specific and unique constraints that are difficult to fully predict and costly to meet on a prototyping budget. Intense consumer-scale miniaturization entails extreme upfront development costs in specialized software, equipment, and labor and is not economical to produce in small volumes. Finally, reliance on hobby-grade packaged modules precludes nearly any miniaturization and presents a rapidly-intolerable combination of both unneeded and missing features. The design compromise resulted in the use of solderable radio modules but otherwise only chip-level integrated packages.

The electronics are powered from a single-cell $(3.7 \mathrm{~V})$ lithium-ion battery, in any parallel combination. Early designs relied on a 3-cell pack storing approximately $36 \mathrm{Wh}$ of energy, while the most recent uses a 1-cell unit storing about $12 \mathrm{Wh}$. Optionally, for data review and programming, USB may be attached externally, with automatic power switchover avoiding unnecessary battery drain. Resultant battery lifetimes depend on the combination of attached sensors, with typical performance figures discussed in Section 5.

Communication - Wireless data communication between nodes and with the parent vehicle is provided by a transceiver on the main PCB. In the prototype system, an XBee transceiver module is selected, implementing the IEEE 802.15.4 Zigbee protocol at $2.4 \mathrm{GHz}$. Use of this module, which integrates the Zigbee protocol stack, provides node discovery and packet hopping, automatically generating a mesh network topology without additional software support in the main microcontroller. The Zigbee protocol was selected over other popular IoT protocols such as WiFi, Bluetooth, or Thread as a compromise offering acceptable range and bandwidth, low power consumption, excellent sleep modes, protocol openness, and simplicity of implementation. In an eventual flight implementation, it is expected and likely that 
a custom transceiver would be designed, optimized for the expected environment in operating frequency, transmit power, and protocol features.

In support of node localization, an additional transceiver measuring inter-node distance (range) is also included. For this purpose, a Decawave Ultra Wideband (UWB) transceiver operating selectively between $3.5 \mathrm{GHz}$ and $6.5 \mathrm{GHz}$ was chosen. Building on recent advances in high speed electronics, these allow estimation of transceiver pair distances using RF time of flight (ToF) measurements at the sub-nanosecond level. The double-sided two-way ranging (DS-TWR) algorithm, as defined by the IEEE 802.15.8-2017 standard [17], was selected. DS-TWR, when compared to other ranging algorithms, such as single-sided two-way ranging (SS-TWR) is advantageous because the extra round of packet exchanges between the two nodes averages out the majority of the clock offset present between the devices, resulting in highly accurate measurements (with as little as $10 \mathrm{~cm}$ quoted by the manufacturer).

Although itself additionally capable of high-speed data transfers, the UWB transceiver was restricted to the task of distance measurement. This provided significant design simplification at the cost of a slightly larger payload and allowed isolated performance characterization without reimplementation of an additional mesh network protocol layer. Future iterations of our design may eliminate the additional transceiver, however this is not currently a priority.

Sensing - Data fusion from multiple onboard sensors is needed to build an effective understanding of the surroundings. We focus primarily on ambient environmental sensors. A modular architecture was selected, with small daughterboards of approximately $1 \times 2 \mathrm{~cm}$ in size, more easily allowing for tailored heterogeneous payload configurations. One such sensor provides common pressure-temperaturehumidity $(\mathrm{PTH})$ readings, and another measures incident light intensity. These contribute foundational data for modeling an area. Additionally, a particulate matter sensor supplies airborne mass density and numeric concentration data, suitable for capturing the dynamic behavior of dust storms. Further, distinct methane and volatile organic compound (VOC) sensors seek to monitor emissions from biological activity such as plant life (in Earth applications) and microbes found in caves. Finally, an inertial measurement unit (IMU) is included for sampling the ballistic trajectories of projectiles in flight, and then for determining projectile orientation once landed. A subset of these sensors is shown in Figure 4.

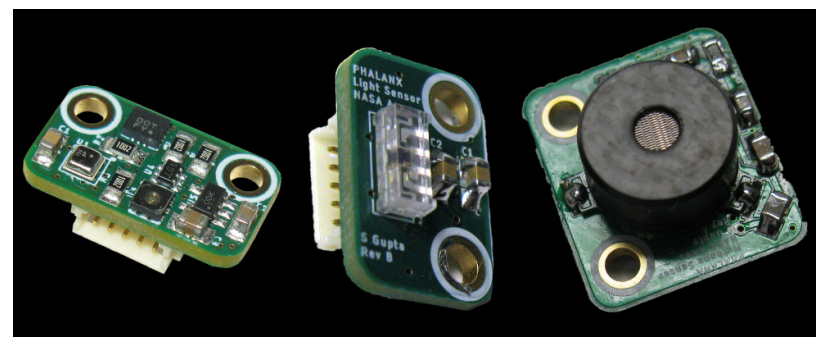

Figure 4. Sensor daughterboards. The major dimension is approximately $2 \mathrm{~cm}$ across.

Given the importance and ubiquitousness of visual sensing, we also consider the task of imaging. In early prototyping, projectiles with a miniature embedded GoPro camera were constructed as a means to evaluate in-flight imaging. Complementary to this, a specific illumination projectile containing high-powered LEDs was also designed, intended to allow a rover's onboard cameras to operate in darkened caves. Example results from these are given in Section 5.

A related path of investigation that became a larger spinoff effort was the development of a miniaturized solid-state 3D microscope suitable for inclusion in a projectile. This consists of a spatially-programmable aperture via a small transmissive LCD and controlled illumination from a ring of LEDs surrounding the lens. This provides stereoscopic imaging and gonioreflectometry of terrain beneath the head of the projectile, without moving parts, allowing high-fidelity characterization of surface materials. The total package fits within a volume of $300 \mathrm{~cm}^{3}$ and weighs less than $150 \mathrm{~g}$, and we are confident that with improved packaging this could be further reduced substantially. The microscope construction and projectile packaging are shown in Figure 5. Full details on its design may be found in a prior publication [18].
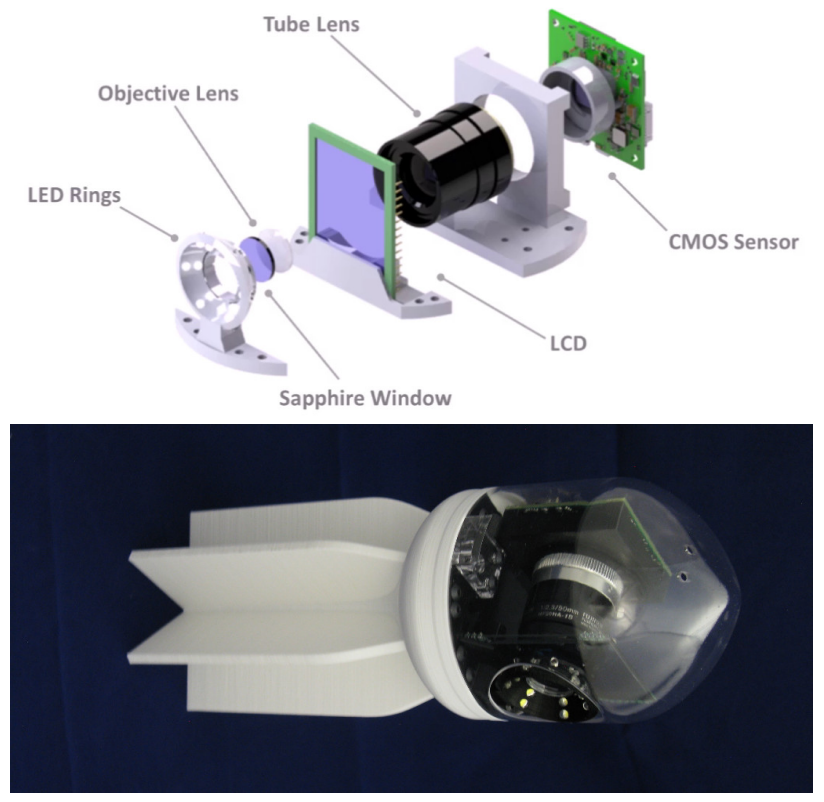

Figure 5. Miniaturized 3D microscope.

Rover-To test our prototype in a realistic scenario, we used KRex2-a four-wheeled rover at NASA Ames used as a development platform for new technologies. It is independently driven and steered. For ground truth, absolute positioning is provided by a GPS-INS system combining differential GPS, a tactical-grade IMU, and wheel odometry offering centimeterlevel accuracy.

The launcher apparatus was mounted to the vehicle, as shown in Figure 6 along with a sensing node intended to represent the central control node electronics that would be integrated with it in a flight mission scenario for data collection and retransmission to Earth. This also represents a platform for testing node mobility, particularly wireless data transfer and range estimation. A moving ranging node allows for reduction or elimination of certain otherwise poorly observable biases in measurements, as it takes readings using the same transceiver from multiple locations. 


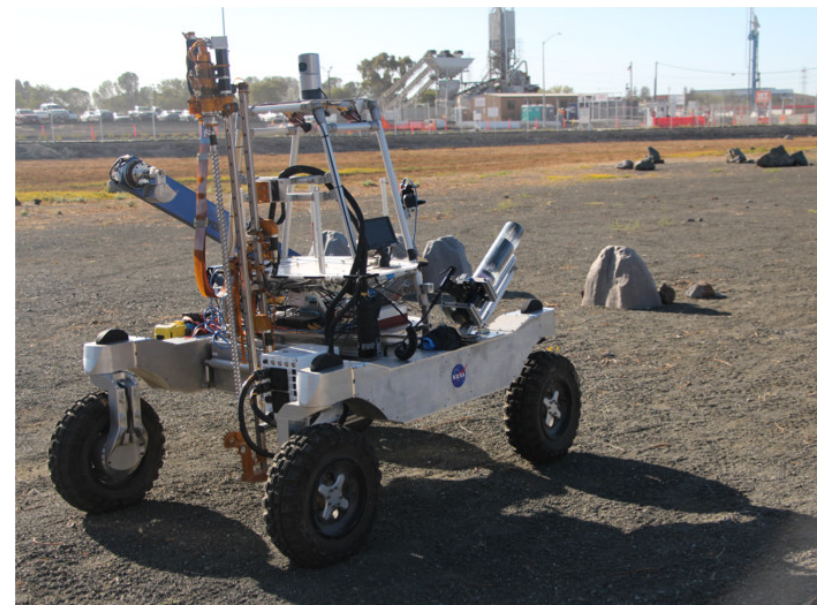

Figure 6. Projectile launcher mounted to KRex2 test rover.

\section{Algorithmic Process}

In operation, a set of nodes is placed at sites within the environment of preferably highest value, the actual resulting location of each node must then be determined, and finally the collected sensor data at each location can be processed to allow interpolation at any other point within the environment. These correspond to three distinct computational problems.

\section{Emplacement Planning}

The performance of a sensor network is significantly influenced by the placement of individual nodes - both relative to terrain features and to each other. For example, nodes that have free lines-of-sight and simultaneous connectivity to many other nodes can increase the overall robustness of the network via multiple routing capability [19]. However, a network that uniformly covers the surface area might produce better sampling over a wide area for science monitoring [20]. These factors are often at odds to one another and mission constraints will further limit the maximum number of nodes that may be available. In our concept of operations, the ability to control ballistic trajectory of projectiles and the characterization of Circular Error Probable, presents opportunities to statistically place these sensors in an advantageous configuration from a remote location. An approach was explored for planning the positions of nodes in the network given some prior information about the terrain in the sensing region. A low-resolution DEM from orbital imagery is a good example of prior data that could be used to assist placement planning.

First, we discuss a method to approximate the RF coverage of a node network sprawled across some arbitrary terrain data-set, where the locations of each node are defined. Such locations may be places of critical interest for obtaining sensor information. Next, an automated placement approach is explored, whereby optimal node locations are computed to provide highest RF coverage over the terrain. It not only enables a network to be constructed from scratch but can be useful for determining the position of intermediary repeater nodes or less critical sensing nodes when a network is already partially established.

Node Placement Metrics-Using sampled heightmap data for a given terrain, we nominate specific locations of interest for nodes to capture sensor information from. With the maximum communication range of our ranging hardware in the tens of meters, nodes should be distributed within comfortable range limits and within line-of-sight to ensure predictable and reliable operation. To enable larger separations, intermediary nodes and routing techniques can be employed to allow them to operate as repeaters.

Multipath propagation can assist in relaying communication to nodes that lack direct line-of-sight by reflecting radio waves from nearby reflective objects. It can however, give rise to Multipath interference, causing degradation of the communication channel and reduction in the accuracy of the time-of-flight ranging system. Reliable predictions of its effects would require highly accurate data-sets of the terrain as well as detailed information about the terrain material and its RF characteristics. Furthermore, with the automation of emplacement planning envisioned to take place on-board a mobile vehicle such as a rover or lander, such computational tasks would be excessive. We therefore do not rely on multipath propagation to enable communication. We also aim to minimize the impact of Multipath interference by ensuring nodes are separated from comparable or larger terrain features, by at least 50 centimetres.

Next,we determine which nodes are within line-of-sight and range of each other, and reports on the completeness of the network, i.e. if all nodes are within range either directly or by routing through one another.

Line-of-sight (LOS): A simple ray casting algorithm is applied over the sampled data-set to ascertain all voxels over the entire map that offer line-of-sight to a given node. Rays are cast from each node at $\left(x_{n}, y_{n}, z_{n}\right)$ and into the $\mathrm{x}-\mathrm{y}$ direction of each voxel around the perimeter. In order for the line-of-sight condition to be met, voxels extending along the ray direction vector must have a height gradient in the $\mathrm{z}$ direction that is either constant or increasing in value upon its previous maximum. Note this method is only valid for surface elevation type data-sets.

Path Loss Approximation: Next, the RF attenuation over distance from the node is computed for all voxels in the map with line-of-sight. The free space path loss $L_{p}$ between a transmitter and receiver having isotropic radiation patterns can be derived from the Friis transmission formula [21] and computed by:

$$
L_{p}=20 \log _{10} \frac{4 \pi d}{\lambda_{0}}
$$

where $d$ is the separation distance between the transmitter and receiver and $\lambda_{0}$ is the free space wavelength at the RF frequency. This formula provides a sufficient approximation for determining communications coverage over our terrain without requiring specific detail of the three-dimensional antenna radiation patterns. Such characteristics would be sensitive to node positioning and orientation as well as to any terrain features that enter the antennas' near-field regions. We consider only the RF coverage of the UWB ranging module, given a maximum communications range of just one quarter of the XBee radio module's range.

The calculation is applied to each and every voxel in the map that presents line-of-sight to each fixed node location, at the RF frequency. Voxels that either lack line-of-sight to any fixed node or to that which exceed the acceptable communications range of our hardware, are identified as dead spots in RF coverage. Lastly, node pairs within line-ofsight and range of each other are identified as well as any 
breakages in the overall network. Manipulation of the node locations may be necessary to extend coverage or enable node communications. Alternatively, additional intermediary nodes may be placed.

Placement Planning Procedure-Starting with one or more fixed node locations, a simple iterative process can be applied to automate the addition of new nodes to a network, in a sequential "next-best" placement approach. The previous line-of-sight and path loss computations are repeated many times over, but with an additional node. The location of the new node is randomly generated for each iteration, with repeat locations ignored. After $\mathrm{N}$ iterations, the node location that provides the highest RF coverage across the terrain while still within communication range of the network, is selected as its optimal position. The same process can then be repeated and further node locations generated to extend the network further. Figure 7 shows an example network expanded to increase RF coverage using this approach.
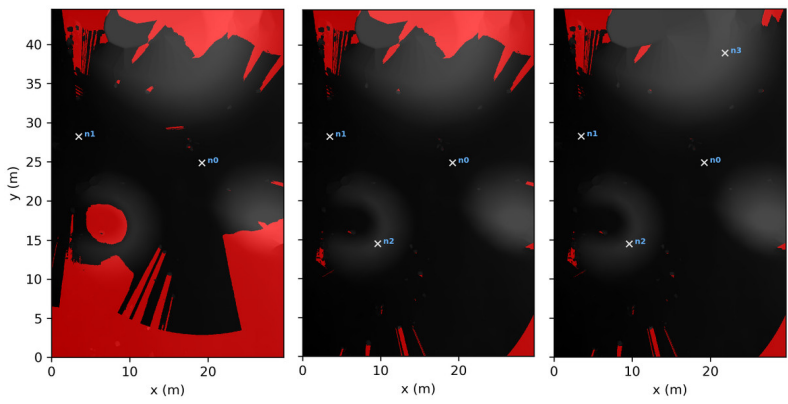

Figure 7. The dead spots in RF coverage, as shown in red, are dramatically reduced as nodes are added to optimized

locations. A communications range of 22 metres was defined, typical of our prototype's $6.5 \mathrm{GHz}$ ranging module.

\section{Network Localization}

Localization of multiple entities such as sensor nodes in an environment is a long and intensely studied problem, with greatly differing approaches depending on even slight nuances in the scenario [20]. Using the distance-measuring transceivers in our prototype, we adopt a range-based approach.

In general, the problem of estimating location from range is called lateration, in contrast to the also commonly encountered (tri-)angulation that estimates location from (angular) bearing measurements. A simplest case to consider is that of trilateration, in which exactly 3 landmarks of known location provide error-free range measurements to a node of unknown location. In this case, the unknown location can be determined analytically, algebraically from the quadratic constraint equations resulting from the measured distances. This may be thought of geometrically in terms of intersecting circles: the unknown node must lie at the intersection of the three circles centered on the respective landmarks and having radius equal to the respective measured distances. Only two landmarks (circles) would result in ambiguity from two possible points of intersection, while one landmark leaves symmetric ambiguity lying anywhere on its surrounding circle.

A generalization of this problem to estimate the location of an unknown node called multi-lateration admits more than three landmarks, as well as range measurements that may be erroneous (e.g. noisy or biased). In this case, the problem is now over-constrained, and a best-fit solution must be found.
This may be performed, for instance, with non-linear least squares optimization [22] as depicted in Figure 8.

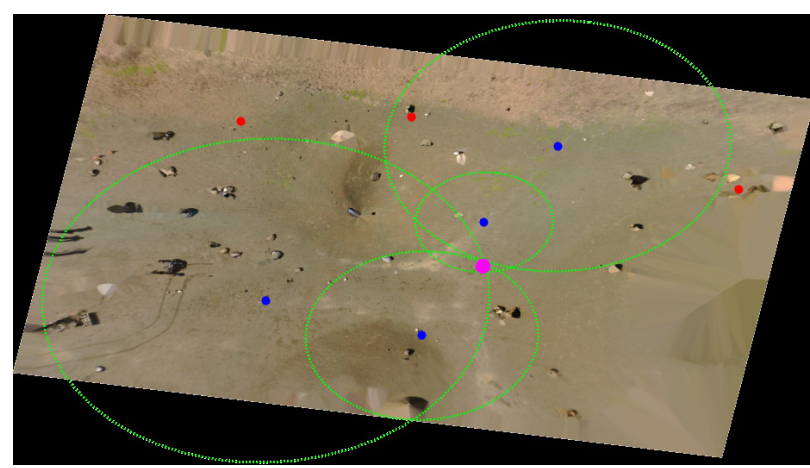

Figure 8. Demonstration of multi-lateration of a single unknown (pink) node location using redundant known locations. Nodes in red are not in contact.

This is essentially the same problem construction as faced in satellite navigation systems such as GPS. As such, one may be reminded that the relative placement of landmarks can have significant effect on the quality of the final estimate, captured in GPS by the quantity referred to as dilution of precision [23]. Geometrically, this may be thought of as layouts that do not adequately eliminate ambiguity in the unknown node's location. An important but trivial case is landmarks that are located extremely close to one another relative to the distance to the unknown node: in this case, the intersecting circles nearly overlap. Another important case is landmarks that are (close to) colinear, represented in Figure 9, which leaves a large near-intersection region within which the estimated unknown location may change greatly with small changes in landmark location or range measurements. These cases leave high uncertainty in the resulting estimate, which may be formalized through error propagation.

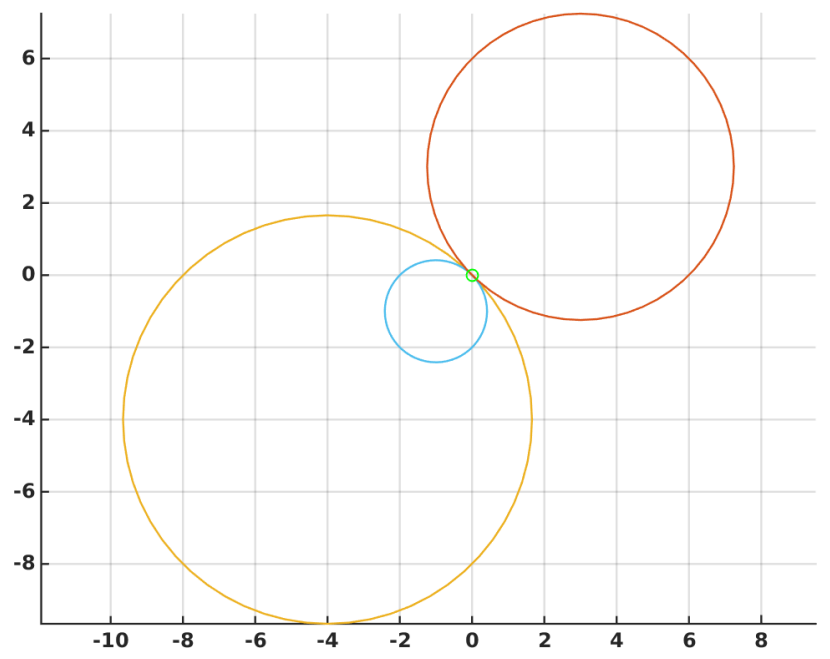

Figure 9. Demonstration of localization sensitivity with co-linear landmarks. Observe the large circle overlap region.

A further generalization of this problem is joint multilateration, corresponding to the broader case in which more than one node's unknown location must be measured from range measurements between nodes, possibly with some number of landmarks. The extreme case is that in which every node has unknown location, corresponding to the basic physical scenario in which multiple nodes are released into 
an environment without awareness of their placement and then begin measuring range to one another. This is therefore our focus. Our solution to this case is to fix an arbitrarily chosen node at an origin location and use measured ranges between triplets of nodes to form triangles, from which the location of the third node in each set may be estimated. Basic information, possibly derived from tracking a rough launch bearing angle on the parent vehicle for each node, is used to disambiguate symmetric flips over coordinate axes. Repeating this for every node results in a seed location estimate that may be then further refined with batch optimization.

Location estimation can also be performed incrementally online, for instance if one or more nodes (such as rovers) are moving. The general version of this problem is range-based simultaneous localization and mapping (SLAM), which may be solved with a large variety of techniques but requires certain caution due to fundamental underlying symmetric radial ambiguity [24].

\section{Sensor Data Mapping}

A common science goal is a continuous, geo-registered map of sensor data, showing its spatial distribution and enabling queries for an estimated sensor reading at any location in the environment, even if a node was not located at that exact point. This can be accomplished by, once node locations are known, smoothly interpolating readings between them.

So far, we have adopted the simple but effective approach of Gaussian mixture interpolation. This estimates the sensor reading at a query location $\mathbf{x}_{q}$ as a distance-weighted average over all nodes, given by

$$
\hat{y}_{q}=\frac{\sum_{i=1}^{N} y_{i} \mathbf{G}\left(\left\|\mathbf{x}_{q}-\mathbf{x}_{i}\right\|, \sigma_{i}^{2}\right)}{\sum_{i=1}^{N} \mathbf{G}\left(\left\|\mathbf{x}_{q}-\mathbf{x}_{i}\right\|, \sigma_{i}^{2}\right)},
$$

where $N$ is the number of nodes, $y_{i}$ is the sensor reading measured by node $i$ located at $\mathbf{x}_{i}, \mathbf{G}\left(d, \sigma^{2}\right)$ is a symmetric Gaussian kernel with standard deviation $\sigma$ evaluated at a radius $d$.

For each node, a value $\sigma_{i}$ must first be chosen, corresponding to a sensing radius for that node. This essentially captures the expected spatial variability of the phenomenon being sensed, or alternatively the distance from a node up to which its reading may be considered applicable.

\section{EXPERIMENTATION AND RESULTS}

\section{Component Characterization}

Power-Measured power draw from key components is given in Table 1. As it shows, total idle power consumption with all sensors disabled lies below $100 \mathrm{~mW}$, offering 5 days of battery life on a single-cell 12Wh battery, or an integer multiple of that for each additional parallel cell. Adding sensors and data transmission reduces this, possibly significantly for e.g. micro-hotplate based gas sensors such as for methane and VOCs, however these may be modulated if required sensing frequency so permits, with duty cycles as low as $10 \%$ quite readily achievable. Further, ranging need not take place continuously for stationary nodes, but only when any node location has changed (e.g. if a node has been added, or the parent rover is moving nearby and requests updates). With such modulation, total power consumption may be reduced

\begin{tabular}{|l|l|}
\hline Component & Power draw [mW] \\
\hline CPU/support electronics & 7.5 \\
\hline UWB ranging transceiver (idle) & 40 \\
\hline UWB ranging transceiver (active) & 550 \\
\hline Zigbee data transceiver (idle) & 50 \\
\hline Zigbee data transceiver (active) & 150 \\
\hline Light/temperature & 0.5 \\
\hline Pressure/temp/humidity & 3 \\
\hline Methane & 160 \\
\hline Particulates (active) & 300 \\
\hline VOC (active) & 200 \\
\hline
\end{tabular}

Table 1. Measured power consumption of respective prototype electronic components.

to still modest levels, possibly at worst halving battery life. It bears further reminder that little power optimization effort has been applied to this design and would receive much greater attention in a production flight version.

Data communications-Even with the low altitude of the Zigbee transceiver antenna above the ground (approximately $5 \mathrm{~cm}$ ), the maximum achievable range for node-to-node (point-to-point) data communications with clear line of sight was measured to be approximately 85 meters, at which point packet loss exceeded $80 \%$. Mesh sizes up to 10 nodes were tested without issue, and per manufacturer documentation, a mesh consisting of up to 30 nodes should remain functional. Further, no limit on the number of hops is imposed, allowing for extremely long chains and resultant physical network diameters, however we enforce a 4 second timeout on packet acknowledgement to prevent hangs. The Zigbee protocol offers a nominal data transfer rate of $250 \mathrm{kbit} / \mathrm{s}$, however due to some packet loss and overheads, we observed only $4.5 \mathrm{kbit} / \mathrm{s}$ effective throughput, up to a maximum tested perhop distance of $20 \mathrm{~m}$. Given the low data volumes and intermittent communication required in our system, even this low bandwidth remains ample.

Ranging-To characterize the accuracy of raw range measurements from the UWB devices, a controlled test was performed in the same test yard used for field experiments, under similar conditions and placing the antennas at similar height above the ground. The two devices had line of sight throughout the test. Results of this experiment are shown in Figure 10. As this shows, even raw readings prior to any joint optimization can be quite accurate, typically within $10 \mathrm{~cm}$ of the correct value, even up to $25 \mathrm{~m}$.

Overall, we found that best ranging performance occurs when the antenna is set vertically upright, is one foot off the ground, and enforce a $1.1 \mathrm{~cm}$ keepout radius for any enclosure material to minimizing reflections. Tilting the antenna induces a slight offset in ranging measurements and reduces the maximum range.

\section{Field Testing}

A variety of single and multiple-node launch and data collection trials were conducted in an outdoor test yard with several 


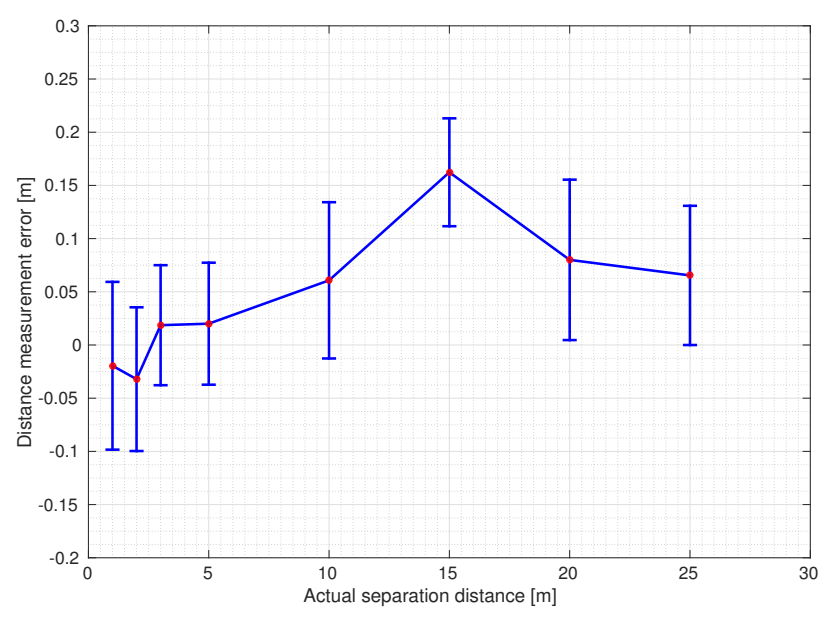

Figure 10. Raw ranging error from two-node UWB distance measurement test. Per-distance averages are based on 100 readings over 30 seconds, and error bars represent 3 standard deviations, showing clustering within approximately $7.5 \mathrm{~cm}$.

gently sloping craters and a surface covered with fine pebbles, providing a rough analog of lunar or Martian terrain.

Mission Scenario-A typical mission rehearsal, depicted in Figure 14, consists of placing 10 nodes within an approximately $20 \times 30 \mathrm{~m}$ area, in and among various terrain obstacles such as small boulders and hills. A mobile surface rover with a node of its own on a short mast moves along an arbitrary trajectory within the environment, opportunistically collecting RF range measurements to reachable nodes for improved localization and then moves away to complete other hypothetical science tasks. After some time, the rover returns to the area to wirelessly retrieve collected sensor data from the network, requiring only communication with a single peripheral node as data is relayed through the network mesh.

Imaging and Illumination-Projectiles containing an onboard video camera were successfully demonstrated as a means of collecting overhead imagery for the purposes of situational awareness. Figure 11 presents a mosaic of 5 frames of recorded video collected from a projectile in flight, giving an "over-the-horizon" perspective of potential obstacles or sites of interest beyond otherwise occluding walls. Image quality is satisfactory despite the use of inexpensive rolling shutter cameras.

At a finer scale, the compact 3D microscope payload for imaging of terrain surfaces from an emplaced projectile was tested on a variety of surface regolith simulants, examples of which are shown in Figure 12, demonstrating its efficacy for material identification and modeling in a miniature remotely emplaced package. For further details of characterization and performance, the reader is referred to the separate publication on this specific payload. [18].

Conversely, prototype illumination-bearing projectiles, shown in Figure 13, were evaluated for the purpose lighting a darkened area, both during flight and once emplaced. While practical for illuminating small areas of enclosed environments such as caves or surface pockets of interest, even the rapidly improving LED illumination efficiencies of 200-300 lumens/Watt require high intensity and a resulting tradeoff of battery size vs. runtime. Depending on the application, this may be somewhat mitigated by an appropriately focused beam or the use of monochromatic illumination concentrating emitted power over a narrower spectrum.

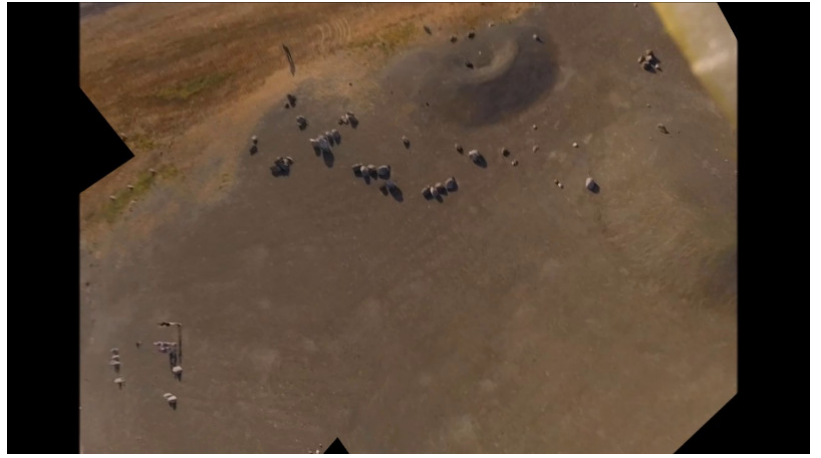

Figure 11. Synthetic overhead view generated from mosaic of images collected while in flight.

Placement Validation - In live testing, the free space path loss model used in the design of the node placement planning concept was evaluated by comparing the set of nodes predicted to be in contact with those that actually were. As shown in Figure 16, the model matches quite closely, with just one false positive (blue dotted line) and negative each (undotted red line).

\section{Conclunsions}

We have introduced a novel exploration payload and mission concept, PHALANX, which pairs expendable projectile sensors with a rover "mothership." Two years of development toward maturing this idea for planetary missions is documented, including mechanism design, selection and miniaturization of COTS electronics, and testing of science sensors. Algorithmic strategies were discussed to emplace the network for optimal science collection as well as provide for post-emplacement localization of the network and exploration robots. Finally, we showed how to produce a spatially interpolated mapping product with point measurements from networked scientific sensors that can be used to address open inquiries in astrobiology.

In field experimentation, we demonstrated a terrestrial prototype with up to 10 nodes, each featuring a complete suite of commodity wireless transceivers, ranging radios, and environmental sensors miniaturized for a $47 \mathrm{~mm}$ diameter projectile. The efficacy of using embedded macro and microimaging sensors to gather relevant scientific data in-flight and post-landing was also indicated. The performance of the communications system was experimentally characterized and found to be effective over inter-node distances of up to 85 meters in planetary terrain, while the UWB ranging system was demonstrated to $+/-10 \mathrm{~cm}$ over 25 meters. Using commodity batteries, nodes were able to last up to 5 days of continuous use with single cell lithium-ion batteries or up to 10x longer on a duty cycle.

We believe the preliminary results discussed here show the potential benefits of using PHALANX in future planetary missions to extend the reach of rovers and conduct longduration, wide-area science. We are also partnering with Earth scientists to tailor the approach to terrestrial monitoring applications. Future work will push toward ever-larger networks ( $>25$ nodes), continued miniaturization (with a goal of $30 \mathrm{~mm}$ diameter projectiles), and end-to-end demonstration including loading from an integrated feed mechanism. We hope to explore performance in-depth against a larger variety of planetary analog terrains and features. 


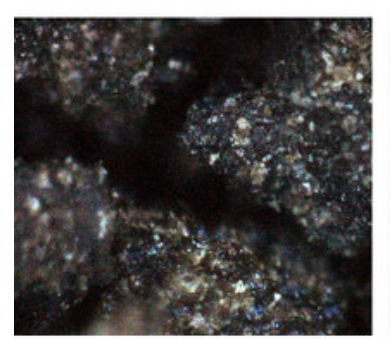

Lunar Mare

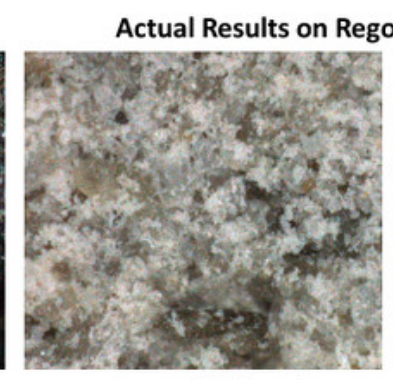

Lunar Highlands

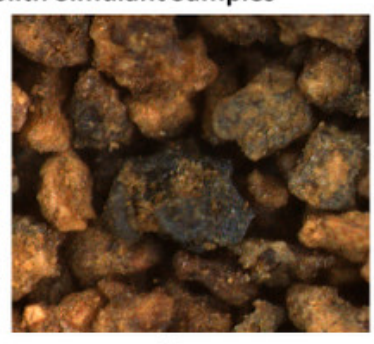

Mars

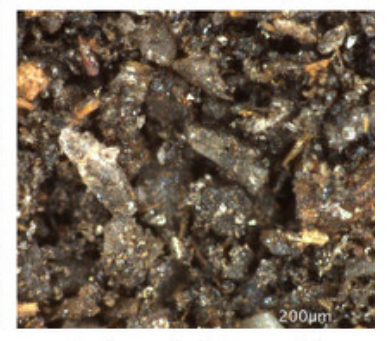

Carbon-rich Asteroid

Figure 12. Image samples recorded from the miniature solid-state 3D microscope on varied samples. Captured at $1 \mu \mathrm{m} / \mathrm{pixel}$.
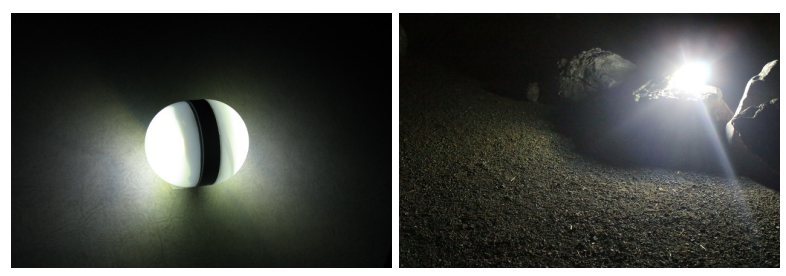

Figure 13. Projectile with illumination payload.

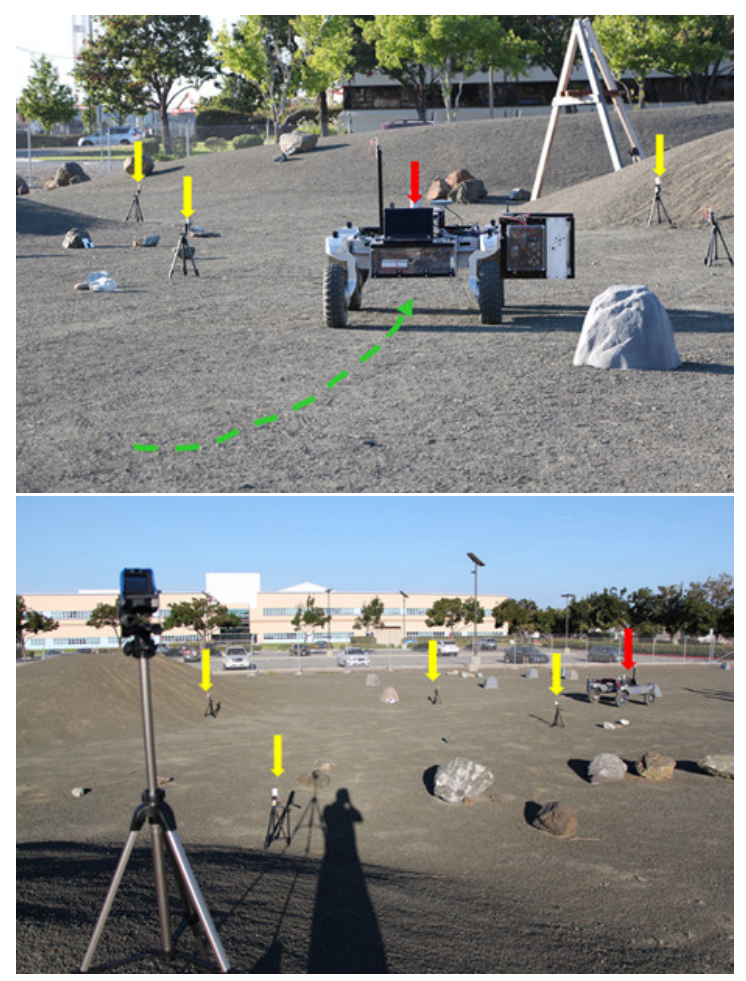

Figure 14. Outdoor multi-node field test setup with mobile rover node. In this particular test, nodes were manually placed on tripods so ground truth positions could be surveyed and range measurements could be isolated from terrain interference.

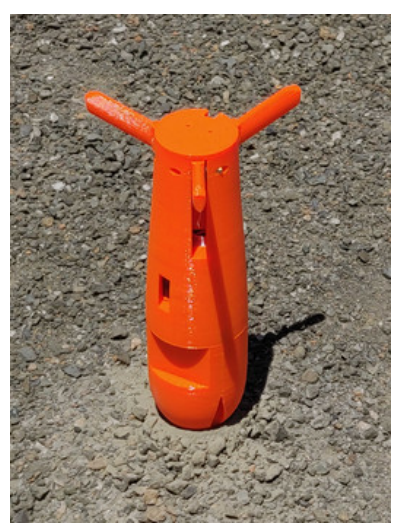

Figure 15. Recent miniaturized dart projectile enclosure emplaced during field test.

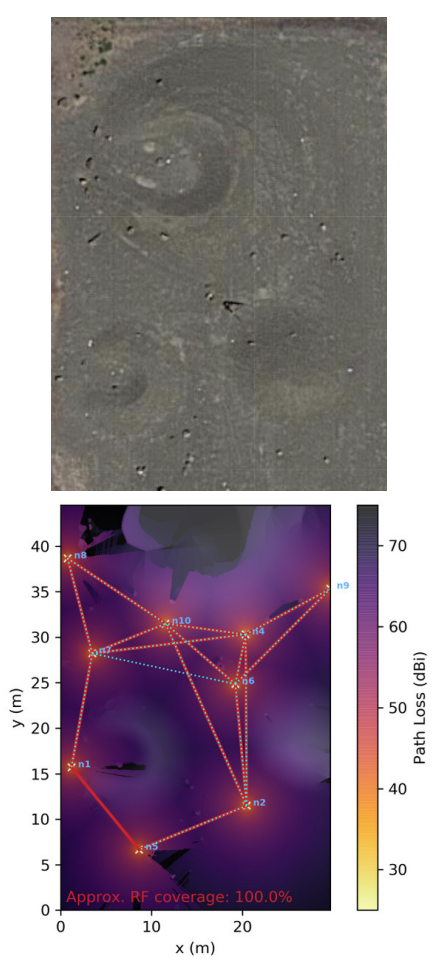

Figure 16. Validation of computed free space path loss. Node pairs that are expected to be within range and line-of-sight are shown by the dotted lines. The solid red lines represent communication links that were established and measured during actual testing.

[Credit: Google, Maxar Technologies] 


\section{ACKNOWLEDGMENTS}

We acknowledge the contributions of Antoine Tardy, Neelay Junnarkar, Arno Rogg, and Eli Zucker. We thank Dr. Steve McGuire and Prof. Nisar Ahmed of the University of Colorado Boulder for many fruitful discussions and for taking on this concept as a class project case study. This project was supported by the NASA Ames Internal Research and Development Fund (IRAD) and the NASA Center Innovation Fund (CIF). We thank NASA SSERVI for use of regolith simulant samples. Danny Nuch was supported by the San Jose State University CAARE Program.

\section{REFERENCES}

[1] D. Reiss-Bubenheim, P. J. Boston, H. Partridge, C. Lindensmith, and J. L. Nadeau, "Seeking the Tricorder: Report on Workshops on Advanced Technologies for Life Detection," AGU Fall Meeting Abstracts, Dec. 2017.

[2] L. Calle, "Corrosion on mars - effects of the mars environment on spacecraft materials," in NASA Technical Publication 220238, 2019.

[3] B. Wilcox, T. Litwin, J. Biesiadecki, J. Matthews, M. Heverly, J. Morrison, J. Townsend, N. Ahmad, A. Sirota, and B. Cooper, "Athlete: A cargo handling and manipulation robot for the moon," in Journal of Field Robotics, vol. 24, 2007, pp. 421-434.

[4] S. Howe, R. O'Brien, N. Jerred, S. Cooley, J. Crepeau, S. Hansen, and A. Klein, "The mars hopper: A radioisotope powered, impulse driven, long-range, long-lived mobile platform for exploration of mars," in Concepts and Approaches for Mars Exploration, 2012.

[5] T. Bretl, S. Rock, J. Latombe, B. Kennedy, and H. Aghazarian, "Free-climbing with a multi-use robot," in Experimental Robotics, vol. IX, 2006, pp. 449-458.

[6] T. A. Liljebäck, P. and K. Fossum, "Serpentine robots for planetary exploration (serpex)," in SINTEF Report, 2014.

[7] B. Balaram, T. Canham, C. Duncan, H. Grip, W. Johnson, J. Maki, A. Quon, R. Stern, and D. Zhu, "Mars helicopter technology demonstrator," in AIAA Atmospheric Flight Mechanics Conference, 2018.

[8] J. Pfeil, K. Hildebrand, C. Gremzow, B. Bickel, and M. Alexa, "Throwable panoramic ball camera," in SIGGRAPH Asia 2011 Emerging Technologies, ser. SA '11. New York, NY, USA: ACM, 2011, pp. 4:1-4:1. [Online]. Available: http://doi.acm.org/10.1145/2073370.2073373

[9] Bounce Imaging Explorer. [Online]. Available: http://www.bounceimaging.com

[10] Panono 360 Camera. [Online]. Available: http://www.panono.com

[11] D. O'Halloran, A. Wolf, and H. Choset, "Design of a high-impact survivable robot," Mechanism and Machine Theory, vol. 40, no. 12, pp. 1345 - 1366, 2005.

[12] A. Parness and C. McKenzie, "Drop: the durable reconnaissance and observation platform," Industrial Robot: An International Journal, vol. 40, no. 3, pp. 218-223, 2013.

[13] P. Birkmeyer, K. Peterson, and R. S. Fearing, "Dash: A dynamic $16 \mathrm{~g}$ hexapedal robot," in 2009 IEEE/RSJ Inter- national Conference on Intelligent Robots and Systems, Oct 2009, pp. 2683-2689.

[14] J. T. Karras, C. L. Fuller, K. C. Carpenter, A. Buscicchio, D. McKeeby, C. J. Norman, C. E. Parcheta, I. Davydychev, and R. S. Fearing, "Pop-up mars rover with textile-enhanced rigid-flex pcb body," in 2017 IEEE International Conference on Robotics and Automation (ICRA), May 2017, pp. 5459-5466.

[15] T. N. Titus, G. E. Cushing, C. Okubo, and R. G. Vaughan, "Wood Valley Pit Crater Cave Microclimate: A Possible Analog for Mars." in 2nd International Planetary Caves Conference, ser. LPI Contributions, vol. 1883, Oct. 2015, p. 9017.

[16] D. Kohanbash, A. Valada, G. Kantor et al., "Base station design and architecture for wireless sensor networks," in Proc. Intl. Conf. Agr. Eng., Valencia, Spain, 2012, pp. $8-12$.

[17] "IEEE standard for wireless medium access control (MAC) and physical layer (PHY) specifications for peer aware communications (PAC)," IEEE Std 802.15.82017, pp. 1-322, Feb 2018.

[18] G. M. Pettersson, M. Dille, S. Abrahamsson, and U. Wong, "Miniature 3D microscope and reflectometer for space exploration," in International Conference on Computational Photography (ICCP), May 2019.

[19] M. Radi, B. Dezfouli, K. A. Bakar, and M. Lee, "Multipath routing in wireless sensor networks: survey and research challenges," sensors, vol. 12, pp. 650-685, 2012.

[20] I. Amundson and X. D. Koutsoukos, "A survey on localization for mobile wireless sensor networks," in International Workshop on Mobile Entity Localization and Tracking in GPS-less Environments. Springer, 2009, pp. 235-254.

[21] S. Haykin and M. Moher, Introduction to analog and digital communications, 2nd ed. John Wiley and Sons, 2007.

[22] W. Murphy and W. Hereman, "Determination of a position in three dimensions using trilateration and approximate distances," Department of Mathematical and Computer Sciences, Colorado School of Mines, Golden, Colorado, MCS-95, vol. 7, p. 19, 1995.

[23] A. Kelly, Mobile robotics: mathematics, models, and methods. Cambridge University Press, 2013.

[24] J. Djugash, "Geolocation with range: Robustness, efficiency and scalability," Ph.D. dissertation, Carnegie Mellon University, Pittsburgh, PA, November 2010. 


\section{BIOGRAPHY}

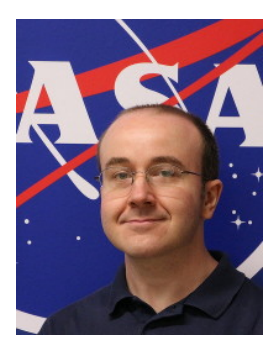

Michael Dille is a senior computer scientist with KBR in the Intelligent Robotics Group at NASA Ames Research Center, where he pursues research in state estimation and novel sensor design. He received his Ph.D. in robotics from Carnegie Mellon University in 2013.

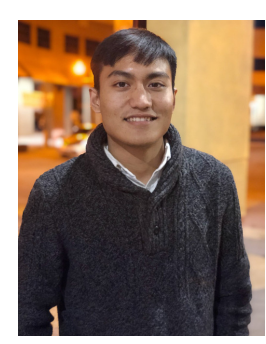

Danny Nuch received his B.S. degree in Computer Engineering at San Jose State University and is continuing for his M.S. degree as a CAARE fellow. He is currently a visiting student researcher with the Intelligent Robotics Group at Nasa Ames Research Center. His research interest lies in the development of autonomous systems.

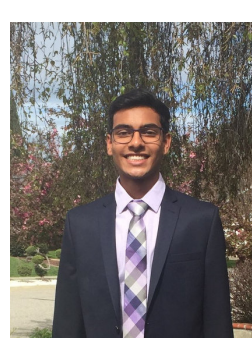

Shiven Gupta is an undergraduate student at Duke University pursuing a degree in Electrical and Computer Engineering. Previously, he was a visiting student researcher at the Intelligent Robotics Group at NASA Ames Research Center. His interests are in robotics and the development of new exploration technologies.

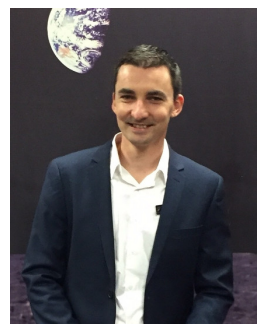

Steven McCabe holds a PhD in Electronic Engineering with the University of Waikato, Hamilton, New Zealand. He completed a post-doctoral student visitorship with the Intelligent Robotics Group at the NASA Ames Research Center, California. His main research interests are in the areas of $R F$ engineering and biomedical systems.

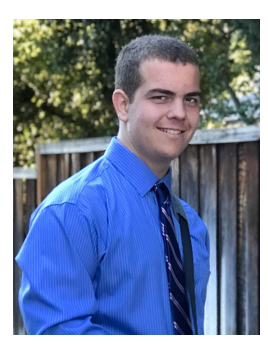

Nicholas Verzic is a visiting student with the Intelligent Robotics Group at NASA Ames Research Center, currently attending the Khan Lab School. He is interested in robotics and artificial intelligence's convergence with economics.

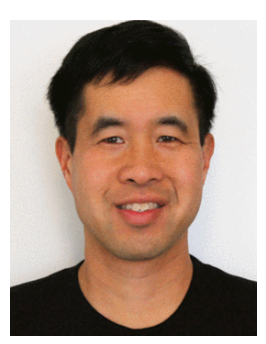

Terry Fong received his B.S. and M.S. degrees in Aeronautics and Astronautics from the Massachusetts Institute of Technology in 1988 and 1990. He received his Ph.D. in Robotics from Carnegie Mellon University in 2001. He is currently the Chief Roboticist at the NASA Ames Research Center. His interests include space robotics, human-robot interaction, and planetary exploration.

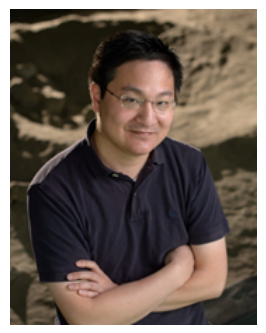

Uland Wong is a senior computer scientist in the Intelligent Robotics Group at the NASA Ames Research Center. His research interest lies at the intersection of computational imaging and mobile robotics. Uland received his Ph.D. in Robotics from Carnegie Mellon in 2012. 